\title{
Approximations for traveltime, slope, curvature, and geometrical spreading of elastic waves in layered transversely isotropic media*
}

\author{
Mohammad Mahdi Abedi ${ }^{1}$, David Pardo ${ }^{2}$, Alexey Stovas ${ }^{3}$ \\ ${ }^{1}$ Basque Center for Applied Mathematics, Bilbao, Spain. E-mail: mabedi@bcamath.org \\ ${ }^{2}$ University of the Basque Country, Department Mathematics, Spain; and Basque Center for \\ Applied Mathematics, Bilbao, Spain; and Ikerbasque, Bilbao, Spain.E-mail: david.pardo@ehu.es \\ ${ }^{3}$ Norwegian university of science and technology Trondheim, Norway. E-mail: \\ Alexey.stovas@ntnu.no
}

\begin{abstract}
Each seismic body wave, including quasi compressional, shear, and converted wave modes, carries useful subsurface information. For processing, imaging, amplitude analysis, and forward modeling of each wave mode, we need approximate equations of traveltime, slope (ray-parameter), and curvature as a function of offset. Considering the large offset coverage of modern seismic acquisitions, we propose new approximations designed to be accurate at zero and infinitely large offsets over layered transversely isotropic media with vertical symmetry axis (VTI). The proposed approximation for traveltime is a modified version of the extended generalized moveout approximation that comprises six parameters. The proposed direct approximations for ray-parameter and curvature use new, algebraically simple, equations with three parameters. We define these parameters for each wave mode without ray tracing so that we have similar approximate equations for all wave modes that only change based on the parameter definitions. However, our approximations are unable to reproduce S-wave triplications that may occur in some strongly anisotropic models. Using our direct approximation of traveltime derivatives, we also obtain a new expression for the relative geometrical spreading. We demonstrate the high accuracy of our approximations using numerical tests on a set of randomly generated multilayer models. Using synthetic data, we present simple applications of our approximations for normal moveout correction and relative geometrical spreading compensation of different wave modes.
\end{abstract}

Keywords: VTI, elastic, moveout, shear wave, converted wave

\section{INTRODUCTION}

Approximations of reflection traveltime, traveltime derivatives, and relative geometrical spreading as a function of offset have applications in ray-based seismic data processing, imaging, amplitude variation with offset (AVO) analysis, and forward modeling. In practice, the application of suitable anisotropic approximations results in enhanced key data processing steps, such as normal moveout correction (Abedi et al, 2019a), time migration (Alkhalifah, 2006; Zhang and Zhang, 2012), and

\footnotetext{
* This paper is publish in GEOPHYSICS 86: C173-C184. https://doi.org/10.1190/geo2021-0215.1
} 
amplitude processing (Alkhalifah, 2011a). To be suitable for modern large offsets acquisition systems, such approximations should be accurate over a wide range of offsets.

Most seismic data processing methods are designed for P-wave data, using the conventional acoustic assumption (Alkhalifah 1998; Alkhalifah 2003); these include multiple traveltime (or moveout) approximations proposed for homogenous or layered anisotropic media (Tsvankin and Thomsen, 1994; Alkhalifah and Tsvankin, 1995; Alkhalifah 2000; Fomel, 2004; Ursin and Stovas, 2006; Aleixo and Schleicher, 2010; Fomel and Stovas, 2010; Alkhalifah, 2011b; Sripanich and Fomel, 2015; Ravve and Koren, 2017; Stovas and Fomel, 2017; Xu et al., 2017; Abedi and Stovas, 2019a, 2019b; Abedi 2020; Abedi and Pardo 2021), and relative geometrical spreading approximations (Ursin and Hokstad, 2003; Stovas and Ursin, 2009; Xu et al., 2019). However, a reflection seismic wavefield consists of different wave modes, including quasi compressional (P), shear (S), and converted (e.g. PS) waves. Each wave mode carries useful subsurface information that is helpful in seismic studies, specifically, in complicated anisotropic environments in which the layers need to be characterized with a larger number of independent parameters.

The conventional acoustic approximation for transversely isotropic (TI) media (Alkhalifah 1998) is a widely accepted simplifying assumption that ignores the effect of the vertical S-wave velocity parameter on P-waves kinematic. However, this parameter enters into the equations that describe $\mathrm{S}$ and converted waves kinematics. Therefore, for applications that use S and PS waves alongside the $\mathrm{P}$-wave data, it is preferable to avoid the acoustic assumption and have uniform elastic equations for $\mathrm{P}, \mathrm{S}$, and the converted waves.

The weak anisotropy approximation (Thomsen, 1986) is another simplifying assumption used for developing traveltime and relative geometrical spreading approximations (e.g. Xu et al., 2005; Farra and Pšenčík, 2013; Farra and Pšenčík, 2019; Farra and Pšenčík, 2020). Traveltime and geometrical spreading approximations for elastic waves that avoid these simplifying assumptions include the methods of Stovas (2010); Hao and Stovas (2016); Xu and Stovas (2019), and Xu et al. (2020), which incorporate model-based parameterization for homogeneous TI models and are used in layered models with ray-traced parameterizations.

We propose explicit direct approximations for traveltime, slope (ray-parameter), curvature, and relative geometrical spreading of elastic waves that are designed for layered TI with vertical symmetry axis (VTI) media. First, we derive the asymptotic series expansions of traveltime, rayparameter, and curvature for P, S, and converted PS waves, at infinite offsets. We identify three effective parameters in the series, with specific definitions for each wave mode. We use these new parameters together with three conventional zero-offset effective parameters in an existing equation for traveltime and our new equations for ray-parameter and curvature. We obtain specific approximations for each wave mode without using the acoustic assumption, weak anisotropy assumption, or ray tracing parameterization. We perform a numerical analysis to compare the errors of our approximations using multiple layered VTI models, and then show a simple application of our approximations in normal moveout (NMO) correction and geometrical spreading compensation of Pand PS-waves.

\section{THEORY}

\section{Series expansions}

To study the kinematic behavior of reflections in layered VTI media, and to obtain fitting constraints for approximating equations over a wide range of offsets, we calculate the Taylor series expansion of 
traveltime squared, ray-parameter, and curvature at zero offset and derive the correct asymptote of each kinematical property at infinite offset.

Using the parametric equations of traveltime and offset (Appendix A), the Taylor series expansion of the traveltime squared around zero-offset reads (Hake et al., 1984; Tsvankin and Thomsen, 1994; Ursin and Stovas, 2006):

$T^{2}(X \rightarrow 0) \approx T_{0}^{2}+\frac{1}{V_{n}^{2}} X^{2}+\frac{1-E_{2}}{4 T_{0}^{2} V_{n}^{4}} X^{4}+\ldots$,

where $T$ is traveltime, $X$ is offset, $T_{0}$ is the effective zero-offset traveltime, $V_{n}$ is the effective normal moveout (NMO) velocity, and $E_{2}$ is the effective second-order anellipticity parameter. Table 1 describes a complete list of symbols used in this work. We use capital letters to denote effective parameters over a stack of layers, and small letters to describe the properties of each individual layer.

At infinitely large offsets, following Abedi and Pardo (2021) and Appendix B, we derive the asymptotic series of traveltime squared as

$T^{2}(X \rightarrow \infty) \approx \frac{1}{\hat{v}_{h}^{2}} X^{2}+\frac{2}{\hat{v}_{h}} E_{\infty} X+\left(E_{\infty}^{2}+\hat{\tau}^{2}\right)+\ldots$,

where $v_{h}$ is the horizontal velocity, $E_{\infty}$ is a function of the properties of all layers, and $\tau$ is the intercept time of the hyperbolic asymptote in one homogeneous layer (Appendix B). The circumflex symbol $(\wedge)$ denotes the properties of the layer with the maximum horizontal velocity in the sequence.

Ray-parameter $(p)$, or the horizontal component of slowness, is given by the first-order derivative of traveltime $(T)$ with respect to offset. The Taylor series expansion of $T$ from zero offset is presented in Ursin and Stovas (2006; equation 22), and the series expansion of $T$ at infinite offsets is presented in Appendix B. To calculate the series expansions of $p$, we calculate the first-order derivative of the series expansions of $T$ as

$$
\begin{aligned}
& p(X \rightarrow 0) \approx \frac{1}{T_{0} V_{n}^{2}} X-\frac{E_{2}}{2 T_{0}^{3} V_{n}^{4}} X^{3}+\ldots, \\
& p(X \rightarrow \infty) \approx \frac{1}{\hat{v}_{h}}-\frac{\hat{\tau}^{2} \hat{v}_{h}}{2 X^{2}}+\ldots
\end{aligned}
$$

Curvature $(\Psi)$ is the second-order derivative of traveltime with respect to offset. Calculating the derivative of the series expansions in equation 3 , we obtain the corresponding series expansions of curvature as

$$
\begin{aligned}
& \Psi(X \rightarrow 0) \approx \frac{1}{T_{0} V_{n}^{2}}-\frac{3 E_{2}}{2 T_{0}^{3} V_{n}^{4}} X^{2}+\ldots, \\
& \Psi(X \rightarrow \infty) \approx \frac{\hat{\tau}^{2} \hat{v_{h}}}{X^{3}}+\ldots
\end{aligned}
$$

\section{Effective parameters}


Six effective parameters appear in the above-calculated series: three well-known zero-offset parameters $\left(T_{0}, V_{n}^{2}\right.$, and $\left.E_{2}\right)$, and three new infinite offsets parameters $\left(E_{\infty}, \hat{v}_{h}\right.$, and $\left.\hat{\tau}\right)$. Among these parameters, $\hat{v_{h}}$, and $\hat{\tau}$ solely depend on the properties of the layer with the maximum horizontal velocity in the sequence. Over a stack of $k$ VTI layers, the zero-offset effective parameters are obtained from the properties of all layers, using the following Dix-type (Dix, 1955) formulas for all wave modes (Tsvankin and Thomsen, 1994; Ursin and Stovas, 2006):

$T_{0}=\sum_{i=1}^{k} t_{0(i)}$

$V_{n}^{2}=T_{0}^{-1} \sum_{i=1}^{k} t_{0(i)} v_{n(i)}^{2}$

$E_{2}=T_{0}^{-1} V_{n}^{-4} \sum_{i=1}^{k} t_{0(i)} \nu_{n(i)}^{4} e_{2(i)}$,

where $t_{0}, v_{n}$, and $e_{2}$ are the zero-offset traveltime, the NMO velocity, and the second-order anellipticity parameter of each layer, respectively. Each of these parameters alongside the infinite offset parameters has a different definition depending on the wave mode. In the following, we define these parameters for P-, S-, and converted PS-waves. A subscripts P, S, or PS, is used to mark the association of the calculated parameters to each wave mode.

\section{P-wave}

For the P-waves, the parameters of each layer used in equation 5 are obtained from the layer properties as (rewritten from Ursin and Stovas, 2006; Stovas, 2010),

$t_{0 P}=2 z / v_{0 P}$,

$v_{n P}^{2}=v_{0 P}^{2}(1+2 \delta)$,

$e_{2 P}=1+\frac{8(\varepsilon-\delta)\left(1+2 \delta-r_{0}^{2}\right)}{(1+2 \delta)^{2}\left(1-r_{0}^{2}\right)}$,

where $r_{0}=v_{0 S} / v_{0 P}, v_{0 P}$ is the vertical P-wave velocity, $v_{0 S}$ is the vertical S-wave velocity, $\varepsilon$ and $\delta$ are the Thomsen (1986) anisotropy parameters, and $z$ is the thickness of each layer. We derive the infinite-offset parameters following Appendix B as,

$$
\begin{aligned}
& \hat{v}_{h P}=\hat{v}_{0 P} \sqrt{1+2 \hat{\varepsilon}}, \\
& \hat{\tau}_{P}^{2}=\left(1+\frac{2(\hat{\varepsilon}-\hat{\delta})\left(1-\hat{r}_{0}^{2}\right)}{1+2 \hat{\delta}-\hat{r}_{0}^{2}+2(\hat{\varepsilon}-\hat{\delta}) \hat{r}_{0}^{2}}\right) \hat{t}_{0 P}^{2}, \\
& E_{\infty P}=\left(\sum_{i \neq M}\left(v_{0 P(i)} t_{0 P(i)} q_{P(i)}\right)\right)_{p=1 / \hat{h}_{h P}},
\end{aligned}
$$

where $q$ is the vertical component of slowness, defined in Appendix B. 


\section{S-wave}

For the S-waves, the parameters of each layer needed in equation 5 (for the calculation of the effective parameters) are a function of the layer properties as (rewritten from Ursin and Stovas, 2006; Stovas, 2010),

$t_{0 S}=2 z / v_{0 S}$,

$v_{n S}^{2}=v_{0 P}^{2}\left(2 \varepsilon-2 \delta+r_{0}^{2}\right)$,

$e_{2 S}=1-\frac{8 r_{0}^{2}(\delta-\varepsilon)\left(1+2 \delta-r_{0}^{2}\right)}{\left(r_{0}^{2}-1\right)\left(2 \varepsilon-2 \delta+r_{0}^{2}\right)^{2}}$.

We derive the infinite-offset parameters following Appendix B as

$$
\begin{aligned}
& \hat{v}_{h S}=\hat{v}_{0 S}, \\
& \hat{\tau}_{S}^{2}=\frac{\hat{r}_{0}^{2}\left(1+2 \hat{\varepsilon}-\hat{r}_{0}^{2}\right) \hat{t}_{0 S}^{2}}{2 \hat{\varepsilon}+\hat{r}_{0}^{2}-\hat{r}_{0}^{4}+2 \hat{\delta}\left(\hat{r}_{0}^{2}-1\right)}, \\
& E_{\infty S}=\left(\sum_{i \neq M}\left(v_{0 S(i)} t_{0 S(i)} q_{S(i)}\right)\right)_{p=1 / \hat{N}_{h S}} .
\end{aligned}
$$

\section{PS-wave}

For the converted PS-waves, the parameters of each layer that are used in equation 5 for the calculation of zero-offset effective parameters are obtained by Dix-type averaging between the incident $\mathrm{P}$ and reflected $\mathrm{S}$ rays as

$$
\begin{aligned}
& t_{0 P S}=\frac{z}{v_{0 P}}+\frac{z}{v_{0 S}}=\frac{t_{0 P}+t_{0 S}}{2}, \\
& v_{n P S}^{2}=\frac{v_{n P}^{2} t_{0 P}+v_{n S}^{2} t_{0 S}}{t_{0 P}+t_{0 S}}, \\
& e_{2 P S}=\frac{t_{0 P} v_{n P}^{4} e_{2 P}+t_{0 S} v_{n S}^{4} e_{2 S}}{\left(t_{0 P}+t_{0 S}\right) v_{n P S}^{2}} .
\end{aligned}
$$

At infinity, the $\mathrm{S}$-wave leg of the converted wave travels a finite distance. Therefore, the parameters of the layer with the maximum horizontal velocity belong to the P-wave. The infinite-offset parameters are calculated following Appendix B as 


$$
\begin{aligned}
& \hat{v}_{h P S}=\hat{v}_{P 0} \sqrt{1+2 \hat{\varepsilon}}, \\
& \hat{\tau}_{P S}^{2}=\left(1+\frac{2(\hat{\varepsilon}-\hat{\delta})\left(1-\hat{r}_{0}^{2}\right)}{1+2 \hat{\delta}-\hat{r}_{0}^{2}+2(\hat{\varepsilon}-\hat{\delta}) \hat{r}_{0}^{2}}\right) \frac{\hat{t}_{0 P}^{2}}{4}, \\
& E_{\infty P S}=\frac{1}{2} E_{\infty P}+\frac{1}{2}\left(\sum_{i}\left(v_{0 S(i)} t_{0 S(i)} q_{S(i)}\right)\right)_{p=1 / \hat{N}_{h P}} .
\end{aligned}
$$

\section{APPROXIMATIONS}

We propose approximate equations for different properties of wave propagation in layered VTI media as explicit functions of offset. These equations are fitted to exact kinematical properties of each wave mode using the terms of the above calculated series as constraints.

\section{Traveltime approximation}

We use the equation designed for traveltime approximation in layered models and introduced by Abedi and Pardo (2021):

$$
T^{2}(X) \simeq T_{0}^{2}+\frac{X^{2}}{V_{n}^{2}}+\frac{A X^{4}}{V_{n}^{4}\left(\sqrt{T_{0}^{4}+2 B X^{2}+C X^{4}}+\sqrt{T_{0}^{4}+D X^{2}}\right)}
$$

This equation is obtained by modifying the extended generalized moveout approximation (EGMA; Abedi and Stovas, 2019b). The key change is the added square root that corrects the form of the asymptote of the equation at infinity for layered media (see Abedi and Pardo, 2021). Equation 12 has six parameters: $T_{0}$, and $V_{n}$, are the zero-offset two-way time and NMO velocity, respectively, which define a background hyperbola (for each wave mode); the remaining four parameters $(A, B, C, D)$ account for the deviations from the background hyperbola. To have a traveltime approximation for each wave mode, we equate the series expansions of equation 12 to the corresponding series in equations 1 and 2 and find the parameters $A, B, C$, and $D$ of equation 12 as a function of model parameters $T_{0}, V_{n}^{2}, E_{2}, E_{\infty}, \hat{v_{h}}$, and $\hat{\tau}$ as follwos:

$$
\begin{aligned}
& A=-\left|\frac{1-E_{2}}{2 \hat{v}_{h}-2 V_{n}}\right|\left(\hat{v}_{h}-V_{n}\right), \\
& B=A^{2} \hat{v}_{h}^{6} \frac{4 E_{\infty}^{2} V_{n}^{2}+\left(\hat{\tau}^{2}-T_{0}^{2}+E_{\infty}^{2}\right)\left(\hat{v}_{h}^{2}-V_{n}^{2}\right)}{V_{n}^{2}\left(\hat{v}_{h}^{2}-V_{n}^{2}\right)^{4}}, \\
& C=\frac{A^{2} \hat{v}_{h}^{4}}{V_{n}^{4}\left(\hat{v}_{h}^{2}-V_{n}^{2}\right)^{2}}, \\
& D=\frac{4 A^{2} E_{\infty}^{2} \hat{v}_{h}^{6}}{\left(\hat{v}_{h}^{2}-V_{n}^{2}\right)^{4}} .
\end{aligned}
$$


For each wave mode, we calculate $T_{0}, V_{n}^{2}$, and $E_{2}$, by inserting equations 6,8 , or 10 into equation 5. We identify the layer with the highest horizontal velocity $\left(\hat{v_{h}}\right)$, and then calculate $E_{\infty}$ and $\hat{\tau}$ from equations 7, 9, or 11. Inserting the calculated $T_{0}, V_{n}^{2}, E_{2}, E_{\infty}, \hat{v_{h}}$, and $\hat{\tau}$ for each wave mode in equation 13, equation 12 approximates the traveltime associated with that wave mode.

In Appendix C, we use a similar process for the equations proposed by Tsvankin and Thomsen (1994), and Ravve and Koren (2017; equation 57) to obtain alternative simpler traveltime approximations for different wave modes. We cannot define all parameters for either the generalized moveout approximation (GMA; Fomel and Stovas, 2010) or EGMA (Abedi and Stovas, 2019b) using a similar strategy because their asymptotes at infinity (available in the original papers) do not include odd powers of $X$.

Figure 1 shows a multilayer VTI model and traced rays for P-, S-, and PS-waves in a common midpoint (CMP) gather. The model properties are presented in Table 2. To show the accuracy of our approximated traveltime for different wave modes and compare it with other methods, we trace rays from the deepest reflector in the model from zero to infinite offsets. Figure 2 shows the relative errors of the approximated traveltime of different wave modes in the presented multilayer model, using different equations. We include the Alkhalifah and Tsvankin (1995) equation with the conventional Dix-type effective parameters in our accuracy comparison. It is the conventional method of traveltime approximation in layered VTI media to use the Dix-type zero-offset effective parameters (equation 5) in three-parameter traveltime equations. We do not include GMA (Fomel and Stovas, 2010) nor EGMA (Abedi and Stovas, 2010) in the numerical comparisons because they have no explicit parameter definition for layered media as a function of the properties of the layers. Both use one traced ray at a finite offset for the definition of their extra parameters. Besides, we cannot use the homogeneous elastic VTI version of GMA (Stovas, 2010) in layered media because no Dix-type averaging is presented for the elastic infinite offset parameters in Stovas (2010). At large offsets, equation 12 is the most accurate approximation for all wave modes, because it has the highest number of fitting constraints at infinite offset.

\section{Ray-parameter approximation}

An explicit approximation of the ray-parameter as a function of offset can be obtained either directly or by taking the derivative of a traveltime approximation. Here, we present both methods.

The first-order derivative of the squareroot of equation 12 and two other traveltime approximations are calculated in Appendix C. Figure 3 shows the accuracy of these moveout-based equations, using the layered VTI model in Table 2.

In layered models, $p$ ranges from 0 to $1 / \hat{v}_{h}$. Although the approximations of $p$ by equations $\mathrm{C}-5$ to C-7 are highly accurate, the value of the approximated $p$ may exceed the maximum value of $p$. Besides, they are algebraically complicated. To avoid these issues, we propose a direct approximation of $p(X)$ using a sigmoid functional form

$$
p(X) \simeq \frac{X}{\sqrt{X^{2} \hat{v}_{h}^{2}+T_{0}^{2} V_{n}^{4}}} .
$$

This simple approximation has the exact value of $p$ and its first-order derivative at zero offset, and the exact value of $p$ at infinite offsets. Figure 3 also shows the accuracy of equation 14. This simple 
three-parameter approximation requires two zero-offset effective parameters $\left(T_{0}, V_{n}^{2}\right)$ and one largeoffset parameter $\left(\hat{v_{h}}\right)$.

\section{Curvature approximation}

An indirect numerical calculation of curvature from the conventional finite difference solutions of the eikonal equation is unstable and rarely usable in practice (Alkhalifah, 2011a). As in the case of the ray-parameter approximations, an explicit analytical approximation of curvature as a function of offset can be obtained either directly or by taking the second-order derivative of a traveltime approximation. The second-order derivative of the square root of equation 12 and two other traveltime approximations are calculated in Appendix C. Figure 4 shows the accuracy of these moveout-based approximations of curvature in a layered VTI model. These equations are algebraically complicated, and the approximated curvature can become negative, even for P-waves. To avoid these issues, we propose the following direct approximation of $\Psi(X)$ :

$$
\Psi(X) \simeq\left(T_{0} V_{n}^{2}+\frac{3 E_{2} X^{2}}{2 T_{0}}\right)^{-1}
$$

This approximation is based on the Taylor series expansion of $\Psi^{-1}(X \rightarrow 0)$. Equation 15 has the exact value of $\Psi$ and its first-order derivative at zero offset, and the exact value of $\Psi$ at infinite offsets (which is zero). Figure 4 also shows the accuracy of equation 15. This equation is more accurate than the indirect ones based on the derivatives of a traveltime approximation (equations $\mathrm{C}$ 8 to $\mathrm{C}-10)$. This simple three-parameter approximation only requires the zero-offset effective parameters $\left(T_{0}, V_{n}^{2}, E_{2}\right)$.

\section{Geometrical spreading approximation}

We use our direct approximations of ray-parameter and curvature in the calculation of relative geometrical spreading as a function of offset. The relative geometrical spreading in horizontally layered media is given by (Ursin and Hokstad, 2003; Stovas and Ursin, 2009),

$$
L=\Omega \sqrt{\frac{X}{p \Psi}},
$$

where $\Omega=\sqrt{\cos \alpha^{r} \cos \alpha^{s}}$ is called the radiation pattern and depends on the cosines of ray-angles $(\alpha)$ for a source-receiver pair. Assuming that the source and the receiver are placed at a horizontal surface and that the first layer is homogeneous, we have $\cos \alpha=z_{(1)} / \sqrt{z_{(1)}^{2}+x_{(1)}^{2}}$ (for more details, see Ursin and Hokstad, 2003). For pure P- or S-waves reflections, we have (Stovas and Ursin, 2009),

$$
\Omega=\frac{v_{0(1)} t_{0(1)}}{\sqrt{v_{0(1)}^{2} t_{0(1)}^{2}+x_{(1)}^{2}}},
$$

and for PS converted reflections we obtain, 
$\Omega=\left(\frac{v_{0 P(1)} t_{0 P(1)} v_{0 S(1)} t_{0 S(1)}}{\sqrt{\left(v_{O P(1)}^{2} t_{0 P(1)}^{2}+x_{P(1)}^{2}\right)\left(v_{O S(1)}^{2} t_{0 S(1)}^{2}+x_{S(1)}^{2}\right)}}\right)^{\frac{1}{2}}$,

where subscript (1) indicates the properties of the first layer. For a reflected ray in a layered model, $x_{(1)}$ is the part of the offset $X$ induced by the ray in layer 1. For each ray in Figure 1, $x_{(1)}$ is seen as the horizontal distance that the ray travels within the first layer.

To approximate geometrical spreading, we follow these steps:

1. Identify the highest horizontal velocity $\left(\hat{v}_{h}\right)$ in the layers above a reflector.

2. Calculate $T_{0}, V_{n}^{2}$, and $E_{2}$, by inserting equations 6,8 , or 10 into equation 5. Alternatively, we can estimate these processing parameters from refelection traveltime using a traveltime approximation.

3. Calculate $p(X)$ from equation 14 , calculate $x_{(1)}$ using equation A-1 for $k=1$, and then calculate the radiation pattern using equation 17 or 18 .

4. Insert $p(X)$ from equation 14 and $\Psi(X)$ from equation 15 into equation 16 , to approximate the relative geometrical spreading as a function of offset $(L(X))$.

\section{Remarks on parameter definitions}

The proposed approximations use parameters of elastic VTI media. Setting the vertical S-wave to Pwave velocity ratio $r_{0}=0$, the calculated series and the proposed approximations reduce to their acoustic version.

The definition of $A$ in equation 13 ensures that $A /\left(\hat{v}_{h}^{2}-V_{n}^{2}\right)<0$. This condition arises from equating the leading term of the series expansion of equation 12 to the corresponding term of the calculated asymptotic series when calculating the parameters in equation 13 .

The classical approximations in equations $\mathrm{C}-1$ and $\mathrm{C}-2$ may result in negative $T^{2}$ at specific offsets when employed for S-wave. The condition $A /\left(\hat{v}_{h}^{2}-V_{n}^{2}\right)<0$ is sufficient to avoid this; therefore, we use the definition of $A$ in equation 13 for these approximations too. A sufficient condition for $A /\left(\hat{v}_{h}^{2}-V_{n}^{2}\right)<0$ is $\varepsilon>\delta$ and $1+2 \delta>r_{0}^{2}$, or $\varepsilon<\delta$ and $1+2 \delta<r_{0}^{2}$ in all layers. If a model satisfies these conditions, the definition of $A$ reduces to: $A=\left(1-E_{2}\right) / 2$.

For the $\mathrm{S}$-waves, the parameter $v_{n S}^{2}$ in equation 8 becomes negative if $2 \varepsilon-2 \delta+r_{0}^{2}<0$. This can potentially result in a negative (or close to zero) effective NMO velocity $\left(V_{n}^{2}\right.$ in equation 5), making any traveltime approximation unstable. To prevent this, we assume $\delta<\varepsilon+r_{0}^{2} / 2$ in all layers. 


\section{NUMERICAL RESULTS}

\section{Accuracy analysis}

To more comprehensively analyze the accuracy of the presented approximations, we calculate their errors in a variety of layered VTI models (similar to the example presented in Figure 2 through 4) and compare their maximum errors. The set consists of 1000 randomly generated models containing two to 14 VTI layers. The layer properties are selected within the predefined range $v_{0 P} \in[2,5] \mathrm{km} / \mathrm{s}$, $r_{0} \in[0.3,0.8], \varepsilon \in[0,0.4], \delta \in[-0.1,0.25], z \in[0.1,0.25] \mathrm{km}$.

Figure 5 compares the maximum relative traveltime errors in the aforementioned set of models, from zero to infinite offsets. Figure 5 employs the same set of models for the P and PS waves; but for the experiment with $\mathrm{S}$-waves, we set the extra conditions $v_{n S}^{2}>0, E_{2}>0$, and also exclude the models that produce triplications since none of the studied approximations can reproduce them. Triplications do not occur when the slowness surface is convex. Figure 5 indicates that equation 12 provides the most accurate results for all wave modes in the majority of models. The higher accuracy of equation 12 is even more pronounced for the PS converted waves.

Figure 6 compares the maximum errors in the approximated ray-parameter (normalized by the maximum value of $p$ ) from zero to infinite offsets using the aforementioned set of layered VTI models. Comparing our direct approximation of ray-parameter in equation 14 with those obtained from taking the derivative of a traveltime approximation, equation 14 is more accurate than equation C- 5 but less accurate than equation C-7 in the majority of the models.

Figure 7 compares the maximum errors in the approximated curvature from zero to infinite offsets, after normalization by the maximum value of $\Psi$, using the same set of layered VTI models as in the previous tests. Our direct approximation of curvature (equation 15) is the most accurate in the majority of the models. Figure 7 shows higher values of maximum errors for the approximation of Swave curvature. This occurs because our experiment includes models that produce sharp changes of curvature at middle offsets, while none of the approximations account for these changes.

\section{Applications}

Using synthetic data, we apply the proposed approximations in NMO correction, and relative geometrical spreading compensation of primary P and PS waves. We consider a layered VTI model (Table 2) where the target reflectors are beneath a layer with the maximum horizontal velocity in the earth model. Assuming that the properties of the high-velocity layer are known, three conventional processing parameters $\left(T_{0}, V_{n}^{2}, E_{2}\right)$ should be estimated for our geometrical spreading approximation, plus a fourth parameter $\left(E_{\infty}\right)$ that should be estimated for NMO correction. In reality, the highvelocity layer can be at any depth, and refraction or more sophisticated walk-away vertical seismic profiling (VSP) studies (e.g. Abedi et al., 2019b) can be used for estimating the high-velocity layer properties.

We generate the synthetic data set for this example using an anisotropic finite-difference modeling of elastic waves. Figure 8a shows a snapshot of the modeled wavefield. Figure $8 \mathrm{~b}$ presents the generated synthetic data. To identify different wave modes in Figure 8b, we also display the traveltimes of $P$ and PS waves obtained by ray tracing (rays are shown in Figure 1). For P-wave reflections, we apply 
an NMO correction using the proposed traveltime approximation (equation 12); we then apply a geometrical spreading correction inserting the proposed ray-parameter and curvature approximations into equation 16. Figure $8 \mathrm{c}$ shows the resulting processed CMP data, where the flat events are the target P-wave primary reflections. Similarly, for PS converted waves, we apply the NMO and geometrical spreading correction using the proposed traveltime, ray-parameter, and curvature approximations. Figure 8d shows the results. Figure 8e and 8f show the separated primary P and PS reflections, using an anisotropic Radon transform (Abedi et al., 2019c). To summarize, Figure 8 shows a CMP processed for P and PS primary reflection data, with potential application for stacking and AVO analysis of each wave mode.

\section{CONCLUSION}

For elastic P, S, and converted PS waves, we derive series expansions of traveltime, slope (rayparameter), and curvature at infinite offset. These series show how the infinite offset asymptote of each kinematical property is dominated by the properties of the layer with the highest horizontal velocity above the reflector. We use three conventional effective parameters for characterizing the truncated Taylor series around the zero offset and define three new effective parameters to explain our asymptotic series at infinite offsets, with explicit definitions based on the layers' properties. Next, we propose approximate equations for traveltime, ray-parameter, and curvature as a function of offset and matched their series expansions -at zero and infinite offsets- to the calculated exact values. As a result, we obtained equations that approximate the kinematics of the different wave modes via a change of parameter definition. All the above-mentioned parameters are used in our traveltime approximation; two zero-offset parameters and one infinite offset parameter are used in our approximation for ray-parameter; and only the three zero-offset parameters are used in our approximation for curvature.

We demonstrate that the errors of our approximations from zero to infinite offsets in one layered VTI model are generally low. We then presente a more comprehensive numerical study using 1000 randomly generated models. Since none of the presented equations approximates the $\mathrm{S}$-wave triplications, we excluded models that produce triplications from our numerical studies on $\mathrm{S}$-wave. Our traveltime approximation and our direct approximation of curvature are the most accurate ones in the majority of the studied models. Our direct approximation of ray-parameter is less accurate than the one based on the derivative of our traveltime approximation, but it is simpler. Our direct approximations of ray-parameter and curvature resulted in a new approximation for geometrical spreading. We show a successful application of the proposed approximations in NMO and geometrical spreading corrections of P and PS waves using synthetic data.

\section{ACKNOWLEDGMENTS}

We are grateful to the journal reviewers, $\mathrm{S}$. $\mathrm{Xu}, \mathrm{J}$. Lu and anonymous reviewers, and to the associate editor T. Alkhalifah, assistant editor A. Guitton and the editor in chief J. Etgen for their insightful comments and suggestions. The first and second authors received funding from the European Union's Horizon 2020 research and innovation program grant agreement no. 777778 (MATHROCKS); the European Regional Development Fund (ERDF) through the Interreg V-A Spain-France-Andorra program POCTEFA 2014-2020 Project PIXIL (EFA362/19); the Spanish Ministry of Science and Innovation with references PID2019-108111RB-I00 (FEDER/AEI) and the "BCAM Severo Ochoa" accreditation of excellence (SEV-2017-0718); and the Basque government through the BERC 20182021 program, the three Elkartek projects 3KIA (KK-2020/00049), EXPERTIA (KK-2021/00048), and SIGZE (KK-2021/00095), and the Consolidated Research Group MATHMODE (IT1294-19) given by the Department of Education. The third author received funding from the GAMES project at NTNU. 


\section{DATA AND MATERIALS AVAILABILITY}

The computer codes that reproduce the numerical analysis presented in this paper are available and can be accessed via the following address:

https://www.mathworks.com/matlabcentral/fileexchange/97954-traveltime-slope-and-curvature-inlayered-vti-media

\section{APPENDIX A}

\section{Parametric equations of traveltime and offset}

For elastic waves (P, S, and converted waves), the parametric equations for exact traveltime $(T)$ and offset $(X)$ over a $k$-layer horizontally layered VTI model, as a function of ray-parameter $(p)$, are (rewritten from Stovas, 2010),

$$
\begin{aligned}
& X(p)=\sum_{i=1}^{k} u_{x(i)} w_{(i)}, \\
& T(p)=\sum_{i=1}^{k} u_{t(i)} w_{(i)},
\end{aligned}
$$

where the index $(i)$ is the layer number, and the terms $u_{x}, u_{t}$, and $w$ are defined for the P-waves, as

$$
\begin{aligned}
& u_{x P}=(1+G+H+2 \delta) t_{0 P} v_{0 P}^{2} p \\
& u_{t P}=\left(1+p^{2} v_{0 P}^{2} H\right) t_{0 P}, \\
& w_{P}=1 / \sqrt{1-(1+G+2 \delta) p^{2} v_{0 P}^{2}}
\end{aligned}
$$

and for the S-waves, as

$$
\begin{aligned}
& u_{x S}=\left(1-G-H+2 \frac{\varepsilon-\delta}{r_{0}^{2}}\right) t_{0 S} r_{0}^{2} v_{0 P}^{2} p, \\
& u_{t S}=\left(1-p^{2} r_{0}^{2} v_{0 P}^{2} H\right) t_{0 S}, \\
& w_{S}=1 / \sqrt{1+\left(2 \delta-2 \varepsilon+(G-1) r_{0}^{2}\right) v_{0 P}^{2} p^{2}} .
\end{aligned}
$$

In the above equations, $G$ and $H$ are the common terms defined as

$$
\begin{aligned}
& H(p)=\frac{G}{\sqrt{Q}}, \\
& G(p)=\frac{4 p^{2}(\varepsilon-\delta)\left(1+2 \delta-r_{0}^{2}\right) v_{0 P}^{2}}{1-\left(r_{0}^{2}-1\right) \sqrt{Q}+2 p^{2} \delta v_{0 P}^{2}-2 p^{2} \varepsilon v_{P 0}^{2}+r_{0}^{2}\left(2 p^{2} \delta v_{0 P}^{2}-1\right)} \\
& Q(p)=1-\frac{4 p^{2}\left(\delta-\varepsilon+\delta r_{0}^{2}\right) v_{0 P}^{2}}{r_{0}^{2}-1}-\frac{4 p^{4}\left(2(\varepsilon-\delta) r_{0}^{2}\left(r_{0}^{2}-1\right)-\left(\varepsilon+\delta\left(r_{0}^{2}-1\right)\right)^{2}\right) v_{0 P}^{4}}{\left(r_{0}^{2}-1\right)^{2}}
\end{aligned}
$$


For converted PS-waves, the exact traveltime and offset are the sum of the traveltime and offset associated with the $\mathrm{P}$ and $\mathrm{S}$ legs of a ray. Since we defined $t_{0 P}$ and $t_{0 S}$ as zero-offset two-way times, we obtain:

$X_{P S}(p)=\frac{1}{2} X_{P}+\frac{1}{2} X_{S}$

$T_{P S}(p)=\frac{1}{2} T_{P}+\frac{1}{2} T_{S}$,

where $X_{P}$ and $X_{S}$ (and similarly $T_{P}$ and $T_{S}$ ) are calculated by inserting equations A-2 and A-3 into equation A-1, respectively.

\section{APPENDIX B}

\section{Definition of infinite offset parameters}

The parametric equations of traveltime and offset for P-, S-, and PS-waves approach infinity when $p \rightarrow 1 / \hat{v}_{h P}, p \rightarrow 1 / \hat{v}_{h S}$, and $p \rightarrow 1 / \hat{v}_{h P}$, respectively. The terms of the series of traveltime squared ( $T^{2}$ ) at infinite offsets have the form $c_{m} X^{m}$ with coefficients (Abedi and Pardo, 2021),

$c_{m}=\lim _{p \rightarrow 1 / \hat{N}_{h}} \frac{T^{2}(p)-\sum_{k=m+1}^{2} c_{k} X^{k}(p)}{X^{m}(p)}$,

where $m=2,1,0, \ldots$. The resulting series of $T^{2}$ is presented in equation 2 .

Following the Abedi and Pardo (2021) method, we derive the series of traveltime $(T)$ at infinite offsets. The terms of the series of $T(X \rightarrow \infty)$ have the form $c_{n} X^{n}$ with coefficients,

$c_{n}=\lim _{p \rightarrow 1 / \hat{v}_{h}} \frac{T(p)-\sum_{k=n+1}^{1} c_{k} X^{k}(p)}{X^{n}(p)}$,

where $n=1,0,-1, \ldots$. Note that the summations in equations B-1 and B-2 are empty for $m=2$ or $n=1$. The resulting series of $T$ reads,

$T(X \rightarrow \infty) \approx \frac{1}{\hat{v}_{h}} X+E_{\infty}+\frac{\hat{\tau}^{2} \hat{v}_{h}}{2} X^{-1}+\ldots$,

In the three leading terms of the above series, three parameters appear: $\hat{v}_{h}$, the maximum horizontal velocity in the sequence, $\hat{\tau}$, the intercept time of the curvilinear asymptote of traveltime squared in the layer with horizontal velocity $\hat{v}_{h}$, and $E_{\infty}$, a new cumulative term in other layers (with time dimension), which we derive in the following. Using the parametric equations for P- or S-waves in Appendix A, and following Abedi and Pardo (2021), $\hat{\tau}$ is obtained by taking the following limit:

$\hat{\tau}=\lim _{p \rightarrow 1 / \hat{\nu}_{h}}\left(\left\{u_{t(M)}^{2}-c_{2} u_{x(M)}^{2}\right\} w_{(M)}^{2}\right)$,

where $M$ is the index of the layer with maximum horizontal velocity $\left(\hat{v_{h}}\right)$. 
Using the parametric equations in Appendix A, and following Abedi and Pardo (2021), we define $E_{\infty}$ for each wave mode as follows:

$$
\begin{aligned}
& E_{\infty P}=\left(u_{x P(M)}^{-1}\left\{u_{x P(M)} \sum_{i \neq M} u_{t P(i)} w_{P(i)}-u_{t P(M)} \sum_{i \neq M} u_{x P(i)} w_{P(i)}\right\}\right)_{p=1 / \hat{v}_{h P}}, \\
& E_{\infty S}=\left(u_{x S(M)}^{-1}\left\{u_{x S(M)} \sum_{i \neq M} u_{t S(i)} w_{S(i)}-u_{t S(M)} \sum_{i \neq M} u_{x S(i)^{w}}{ }_{S(i)}\right\}\right)_{p=1 / \hat{v}_{h S}}, \\
& E_{\infty P S}=\left(u_{x P(M)}^{-1}\left\{u_{x P(M)} \sum_{i \neq M} u_{t P(i)} w_{P(i)} \sum_{i} u_{t S(i)^{w}} w_{S(i)}-u_{t P(M)} \sum_{i \neq M} u_{x P(i)} w_{P(i)} \sum_{i} u_{x S(i)} w_{S(i)}\right\}\right)_{p=1 / \hat{w}_{h P}} .
\end{aligned}
$$

Since $u_{x(M)}^{-1} u_{t(M)}=1 / \hat{v}_{h}$, these equations simplify to,

$$
\begin{aligned}
& E_{\infty P}=\left(\sum_{i \neq M}\left(u_{t P(i)^{w}} w_{P(i)}-u_{x P(i)} w_{P(i)} p\right)\right)_{p=1 / \hat{v}_{h P}}, \\
& E_{\infty S}=\left(\sum_{i \neq M}\left(u_{t S(i)^{w}} w_{S(i)}-u_{x S(i)^{w}}{ }_{S(i)} p\right)\right)_{p=1 \hat{N}_{h S}}, \\
& E_{\infty P S}=\left(\sum_{i \neq M}\left(u_{t P(i)} w_{P(i)}-u_{x P(i)} w_{P(i)} p\right)+\sum_{i}\left(u_{t S(i)} w_{S(i)}-u_{x S(i)} w_{S(i)} p\right)\right)_{p=1 / \hat{v}_{h P}} .
\end{aligned}
$$

We have

$$
u_{t(i)} \mathcal{w}_{(i)}=v_{0(i)} t_{0(i)} q_{(i)}+u_{x(i)} w_{(i)} p
$$

where for $\mathrm{P}$ and $\mathrm{S}$ waves, $v_{0}$ is the vertical velocity, and $q(p)$ is the vertical component of slowness, given by

$$
\begin{aligned}
& q(p)=\sqrt{\frac{g_{1} \pm \sqrt{g_{1}^{2}-g_{2}}}{2 r_{0}^{2} v_{0 P}^{2}}}, \\
& g_{1}=1+r_{0}^{2}-2\left(\varepsilon+(1+\delta) r_{0}^{2}-\delta\right) v_{0 P}^{2} p^{2}, \\
& g_{2}=4 r_{0}^{2}\left((1+2 \varepsilon) v_{0 P}^{2} p^{2}-1\right)\left(p^{2} r_{0}^{2} v_{0 P}^{2}-1\right) .
\end{aligned}
$$

The vertical slowness of $\mathrm{P}$ - and $\mathrm{S}$-waves $\left(q_{P}\right.$ and $\left.q_{S}\right)$ are obtained by using the plus and minus signs in equation B-12, respectively. Using equation B-11, equations B-8 through B-10 further simplify to

$$
\begin{aligned}
& E_{\infty P}=\left(\sum_{i \neq M}\left(v_{0 P(i)} t_{0 P(i)} q_{P(i)}\right)\right)_{p=1 / \hat{v}_{h P}}, \\
& E_{\infty S S}=\left(\sum_{i \neq M}\left(v_{0 S(i)} t_{0 S(i)} q_{S(i)}\right)\right)_{p=1 / \hat{v_{h S}}},
\end{aligned}
$$




$$
E_{\infty P S}=\frac{1}{2} E_{\infty P}+\frac{1}{2}\left(\sum_{i}\left(v_{0 S(i)} t_{0 S(i)} q_{S(i)}\right)\right)_{p=1 / \hat{v}_{h P}} .
$$

\section{APPENDIX C}

\section{Traveltime approximations and their derivatives}

In addition to the traveltime approximation in equation 12, we consider two traveltime approximations with modified parameter definitions for asymptotic correction in layered media. We then calculate the first and second-order derivatives of the three of them with respect to offset.

The traveltime approximation by Tsvankin and Thomsen (1994) reads

$$
T^{2}(X) \simeq T_{0}^{2}+\frac{X^{2}}{V_{n}^{2}}+\frac{A X^{4}}{V_{n}^{4}\left(T_{0}^{2}+B_{H} \frac{X^{2}}{V_{n}^{2}}\right)},
$$

where $T_{0}, V_{n}$, and $A$ have the same definition used for equation 12, and $B_{H}$ is obtained after the asymptotic correction as (Ravve and Koren, 2017; Abedi and Pardo, 2021)

$$
B_{H}=\frac{A \hat{v}_{h}^{2}}{V_{n}^{2}-\hat{v}_{h}^{2}}
$$

The Ravve and Koren (2017; equation 57) approximation reads

$$
T^{2}(X) \simeq T_{0}^{2}+\frac{X^{2}}{V_{n}^{2}}+\frac{A X^{4}}{V_{n}^{4}\left(B_{H} \frac{X^{2}}{V_{n}^{2}}+\sqrt{T_{0}^{4}+2 B_{L} \frac{T_{0}^{2} X^{2}}{V_{n}^{2}}}\right)}
$$

The parameter $B_{L}$ in equation C-2 is defined by Abedi and Pardo (2021) as

$$
B_{L}=\frac{4 A^{2} E_{\infty}^{2} \hat{v}_{h} V_{n}^{2}}{T_{0}^{2}\left(\hat{v}_{h}^{2}-V_{n}^{2}\right)^{4}}
$$

The first-order derivative of the square root of equation C-1 reads

$$
p(X) \simeq \frac{X}{T V_{n}^{2}}\left(1+\frac{A\left(B_{H} X^{4}+2 X^{2} T_{0}^{2} V_{n}^{2}\right)}{\left(B_{H} X^{2}+T_{0}^{2} V_{n}^{2}\right)^{2}}\right),
$$

where $T$ is the traveltime approximation in equation $\mathrm{C}-1$.

The first-order derivative of the square root of equation C-3 reads 
$p(X) \simeq \frac{X^{5}\left(2 B_{H}\left(A+B_{H}\right) R_{3}-A B_{L} T_{0}^{2}\right)+4 X^{3}\left(A+B_{H}\right) R_{3}^{2} V_{n}^{2}+2 X R_{3}^{3} V_{n}^{4}}{2 T R_{3}\left(X^{2} B_{H} V_{n}+R_{3} V_{n}^{3}\right)^{2}}$,

where $T$ is the traveltime approximation in equation C-3, and $R_{3}=\sqrt{T_{0}^{4}+2 B_{L} \frac{T_{0}^{2} X^{2}}{V_{n}^{2}}}$.

Likewise, the first-order derivative of the square root of equation 12 reads

$$
p(X) \simeq \frac{X}{2 T V_{n}^{4}}\left(2 V_{n}^{2}+\frac{4 A X^{2}}{R_{1}+R_{2}}-\frac{2 A X^{4}}{\left(R_{1}+R_{2}\right)^{2}}\left(\frac{B+C X^{2}}{R_{1}}+\frac{D}{2 R_{2}}\right)\right)
$$

where $T$ is the traveltime approximation in equation $12, R_{1}=\sqrt{T_{0}^{4}+2 B X^{2}+C X^{4}}$ and $R_{2}=\sqrt{T_{0}^{4}+D X^{2}}$.

The second-order derivative of the square root of equation C-1 reads

$$
\begin{aligned}
\Psi(X) & \simeq-\frac{p^{2}}{T}+ \\
& \frac{B^{2}(A+B) X^{6}+3 B(A+B) X^{4} T_{0}^{2} V_{n}^{2}+3(2 A+B) X^{2} T_{0}^{4} V_{n}^{4}+T_{0}^{6} V_{n}^{6}}{T V_{n}^{2}\left(B X^{2}+T_{0}^{2} V_{n}^{2}\right)^{3}}
\end{aligned}
$$

where $T$ is replaced with the square root of equation $\mathrm{C}-1$, and $p$ is replaced with equation C-5.

The second-order derivative of the square root of equation C-3 reads

$$
\begin{aligned}
\Psi(X) \simeq-\frac{p^{2}}{T}+\frac{1}{T V_{n}^{2}}+\frac{A B_{H}\left(6 X^{8} B_{L}^{2} T_{0}^{2}+11 X^{6} B_{L} T_{0}^{4} V_{n}^{2}+6 X^{4} T_{0}^{6} V_{n}^{4}\right)}{2 T R_{3} V_{n}^{2}\left(X^{2} B_{H}+R_{3} V_{n}^{2}\right)^{3}\left(X^{2} B_{L}+T_{0}^{2} V_{n}^{2}\right)}+ \\
\frac{A X^{2}\left(2 X^{6} B_{H}^{2} B_{L}+2 X^{4}\left(B_{H}^{2}+3 B_{L}^{2}\right) T_{0}^{2} V_{n}^{2}+15 X^{2} B_{L} T_{0}^{4} V_{n}^{4}+12 T_{0}^{6} V_{n}^{6}\right)}{2 T V_{n}^{2}\left(X^{2} B_{H}+R_{3} V_{n}^{2}\right)^{3}\left(X^{2} B_{L}+T_{0}^{2} V_{n}^{2}\right)},
\end{aligned}
$$

where $T$ is replaced with the square root of equation C-3, and $p$ is replaced with equation C-6. The second-order derivative of the square root of equation 12 reads

$$
\begin{aligned}
\Psi(X) \simeq \frac{1}{T}\left(\frac{1}{V_{n}^{2}}-p^{2}-\frac{2 A X^{2}\left(2 X R_{4}-3 R_{5}\right)}{R_{5}^{2} V_{n}^{4}}\right)+ \\
A X^{4} \frac{2 R_{4}^{2}-R_{5}\left(\frac{D\left(-D X^{2}+R_{2}^{2}\right)}{R_{2}^{3}}+\frac{2\left(C X^{4}\left(3 B+C X^{2}\right)+\left(B+3 C X^{2}\right) T_{0}^{4}\right)}{R_{1}^{3}}\right)}{2 R_{5}^{3} V_{n}^{4} T},
\end{aligned}
$$


where $T$ is replaced with the square root of equation $12, p$ is replaced with equation C-7, $R_{4}=\left(\frac{2 X\left(B+C X^{2}\right)}{R_{1}}+\frac{D X}{R_{2}}\right)$, and $R_{5}=R_{1}+R_{2}$.

\section{REFERENCES}

Abedi, M.M., 2020, Rational approximation of P-wave kinematics - Part 1: Transversely isotropic media: Geophysics, 85 no.5, C163-C173.

Abedi, M.M. and D., Pardo, 2021, Large-offset P-wave traveltime in layered transversely isotropic media: Geophysics, 86 no.3, C65-C74.

Abedi M.M., M.A. Riahi, and A. Stovas, 2019a, Three-parameter NMO correction in layered anisotropic media: a stretch-free approach: Geophysics, 84, no. 3, C129-C142.

Abedi, M. M., M. Rashidi Fard, M. A. Riahi, 2019b, Estimation of in situ anisotropy parameters from two perpendicular walkaway VSP lines in South Pars field: Journal of Geophysics and Engineering, 16, 926-938.

Abedi, M. M., M. A. Riahi, and A. Stovas, 2019c, Three-parameter Radon transform in layered transversely isotropic media: Geophysical Prospecting, 67, 395-407, doi: 10.1111/13652478.12731.

Abedi, M.M., and A. Stovas, 2019a, A new parameterization for generalized moveout approximation, based on three rays: Geophysical Prospecting, 67, no.5, 1243-1255. DOI:10.1111/13652478.12770

Abedi, M.M., and A. Stovas, 2019b, Extended generalized nonhyperbolic moveout approximation: Geophysical Journal International, 216, 1428-1440.

Aleixo R. and J. Schleicher, 2010, Traveltime approximations for $\mathrm{qP}$ waves in vertical transversely isotropy media: Geophysical Prospecting, 58 no. 2, 191-201.

Alkhalifah, T., 1997, Velocity analysis using nonhyperbolic moveout in transversely isotropic media: Geophysics, 62, 1839-1854.

Alkhalifah, T., 1998, Acoustic approximations for processing in transversely isotropic media: Geophysics, 63, 623-631.

Alkhalifah T., 2000, The offset-midpoint traveltime pyramid in transversely isotropic media: Geophysics, 65, 1316-25.

Alkhalifah, T., 2003, An acoustic wave equation for orthorhombic anisotropy: Geophysics, 68, 11691172, doi: 10.1190/1.1598109.

Alkhalifah, T., 2006, Kirchhoff time migration for transversely isotropic media: An application to Trinidad data: Geophysics, 71, no. 1, S29-S35.

Alkhalifah, T., 2011a, Efficient traveltime compression for 3D prestack Kirchhoff migration: Geophysical Prospecting, 59, 1-9.

Alkhalifah T., 2011b, Scanning anisotropy parameters in complex media: Geophysics 76, no. 2, U1322.

Alkhalifah T., and I. Tsvankin, 1995, Velocity analysis for transversely isotropic media: Geophysics, 60, 1550-1566.

Dix, C. H., 1955, Seismic velocities from surface measurements: Geophysics, 20, 68-86, doi: 10.1190/1.1438126.

Farra, V. and I. Pšenčík, 2013, Moveout approximations for P-and SV-waves in VTI media: Geophysics, 78, no.5, WC81-WC92.

Farra, V. and I. Pšenčík, 2019, Reflection moveout approximation for a P-SV wave in a moderately anisotropic homogeneous vertical transverse isotropic layer: Geophysics, 84, no.2, C75-C83. 
Farra, V. and I. Pšenčík, 2020, P-wave reflection-moveout approximation for horizontally layered media of arbitrary moderate anisotropy: Geophysics, 85, no. 2, C61-C70.

Fomel S., 2004, On anelliptic approximations for qP velocities in VTI media: Geophysical Prospecting, 52, no.3, 247-259.

Fomel, S., and A. Stovas, 2010, Generalized nonhyperbolic moveout approximation: Geophysics, 75, no. 2, U9-U18.

Hake, H., K. Helbig, and C.S., Mesdag, 1984, Three-term Taylor series for t2-x2-curves of P-and Swaves over layered transversely isotropic ground: Geophysical Prospecting, 32 no.5, 828-850.

Hao, Q. and A. Stovas, 2016, Generalized moveout approximation for P-SV converted waves in vertically inhomogeneous transversely isotropic media with a vertical symmetry axis: Geophysical Prospecting, 64, no. 6,1469-1482.

Ravve I., and Z. Koren, 2017, Traveltime approximation in vertical transversely isotropic layered media, Geophysical Prospecting, 65 no.6, 1559-1581.

Stovas, A., 2010, Generalized moveout approximation for $\mathrm{qP}$-and $\mathrm{qSV}$-waves in a homogeneous transversely isotropic medium: Geophysics, 75 no.6, D79-D84.

Stovas A., and S. Fomel, 2017, The modified generalized moveout approximation: a new parameter selection: Geophysical Prospecting, 65 no. 3, 687-695.

Stovas, A. and B., Ursin, 2009, Improved geometric-spreading approximation in layered transversely isotropic media: Geophysics, 74 no.5, D85-D95.

Sripanich, Y., and S. Fomel, 2015, On anelliptic approximations for qP velocities in transversely isotropic and orthorhombic media: Geophysics, 80 no. 5, C89-C105, doi: 10.1190/geo20140534.1.

Thomsen, L., 1986, Weak elastic anisotropy: Geophysics, 51, 1954-1966.

Tsvankin I., and L. Thomsen, 1994, Nonhyperbolic reflection moveout in anisotropic media: Geophysics, 59, 1290-1304.

Ursin, B., and K. Hokstad, 2003, Geometrical spreading in a layered transversely isotropic medium with vertical symmetry axis: Geophysics, 68,2082-2091.

Ursin B., and A. Stovas, 2006, Traveltime approximations for a layered transversely isotropic medium. Geophysics, 71 no.2, D23-33.

$\mathrm{Xu}$, S., and A. Stovas, 2018, Generalized non-hyperbolic approximation for $\mathrm{qP}$-wave relative geometrical spreading in a layered transversely isotropic medium with a vertical symmetry axis: Geophysical Prospecting, 66, 1290-1302.

Xu, S., and A. Stovas, 2019, Traveltime approximation for converted waves in elastic orthorhombic media, Geophysics, 84, C229-C237.

$\mathrm{Xu}$, S., A. Stovas, and Q., Hao, 2017, Perturbation-based moveout approximations in anisotropic media: Geophysical Prospecting, 65, no.5, 1218-1230.

$\mathrm{Xu}$, S., Stovas, A. and H., Mikada, 2020, Traveltime and relative geometric spreading approximation in elastic orthorhombic medium: Geophysics, 85, no.5, C153-C162.

Xu, X., I. Tsvankin, and A. Pech, 2005, Geometrical spreading of P-waves in horizontally layered, azimuthally anisotropic media: Geophysics, 70, no.5, D43-D53.

Zhang, J. and J. Zhang, 2012, Amplitude-preserved pre-stack time migration in 3D VTI media: Exploration Geophysics, 43, 171-177. 
Table 1. List of symbols and their definitions. Adding P, S, or PS subscripts indicates the association of a parameter to that wave mode. Adding a circumflex over a parameter means the parameter belongs to the layer with the maximum horizontal velocity in the sequence.

\begin{tabular}{ll}
\hline Symbol & Definition \\
\hline$A, B, C, D$ & Parameters of equation 12, defined in equation 13 \\
\hline$e_{2}, E_{2}{ }^{*}$ & Second-order anellipticity \\
\hline$E_{\infty}$ & An infinite-offset parameter defined in equations 7, 9, and 11 \\
\hline$L$ & Relative geometrical spreading \\
\hline$M$ & Index of the layer with the maximum horizontal velocity \\
\hline$p$ & Ray parameter, slope, or horizontal component of slowness \\
\hline$q$ & The vertical component of slowness \\
\hline$r_{0}$ & The ratio of vertical velocities of S-wave to P-wave \\
\hline$t, T^{*}$ & Traveltime \\
\hline$t_{0}, T_{0}{ }^{*}$ & Zero-offset two-way traveltime \\
\hline$v_{0}$ & Vertical velocity \\
\hline$v_{h}$ & Horizontal velocity \\
\hline$v_{n}, V_{n}{ }^{*}$ & NMO velocity \\
\hline$x, X^{*}$ & Offset \\
\hline$z$ & The thickness of a layer \\
\hline$\delta, \varepsilon$ & Anisotropy parameters \\
\hline$\tau$ & An infinite-offset parameter defined in equations 7, 9, and 11 \\
\hline$\Psi$ & Curvature \\
\hline$*^{*}$ & Radiation pattern \\
\hline
\end{tabular}

*The small letters are associated with one homogenous layer and the capital letters are associated with a stack of layers.

Table 2. Properties of the layered VTI models used in Figure 1, 2, 3, 4, and 8. Note that the ordering of the layers does not affect the errors of the kinematical properties presented in Figures 2 to 4.

\begin{tabular}{cccccc}
\hline Layer number & $v_{0 P}(\mathbf{k m} / \mathbf{s})$ & $r_{0}$ & $\varepsilon$ & $\delta$ & $\boldsymbol{z}(\mathbf{k m})$ \\
\hline $\mathbf{1}$ & 3.0 & 0.673 & 0 & -0.040 & 0.3 \\
\hline $\mathbf{2}$ & 2.7 & 0.579 & 0.37 & -0.005 & 0.3 \\
\hline $\mathbf{3}$ & 2.2 & 0.577 & 0.07 & 0.030 & 0.3 \\
\hline $\mathbf{4}$ & 2.5 & 0.580 & 0.11 & 0.065 & 0.2 \\
$\mathbf{5}$ & 2.0 & 0.575 & 0.15 & 0.100 & 0.3 \\
\hline
\end{tabular}


Elastic approximations for layered media
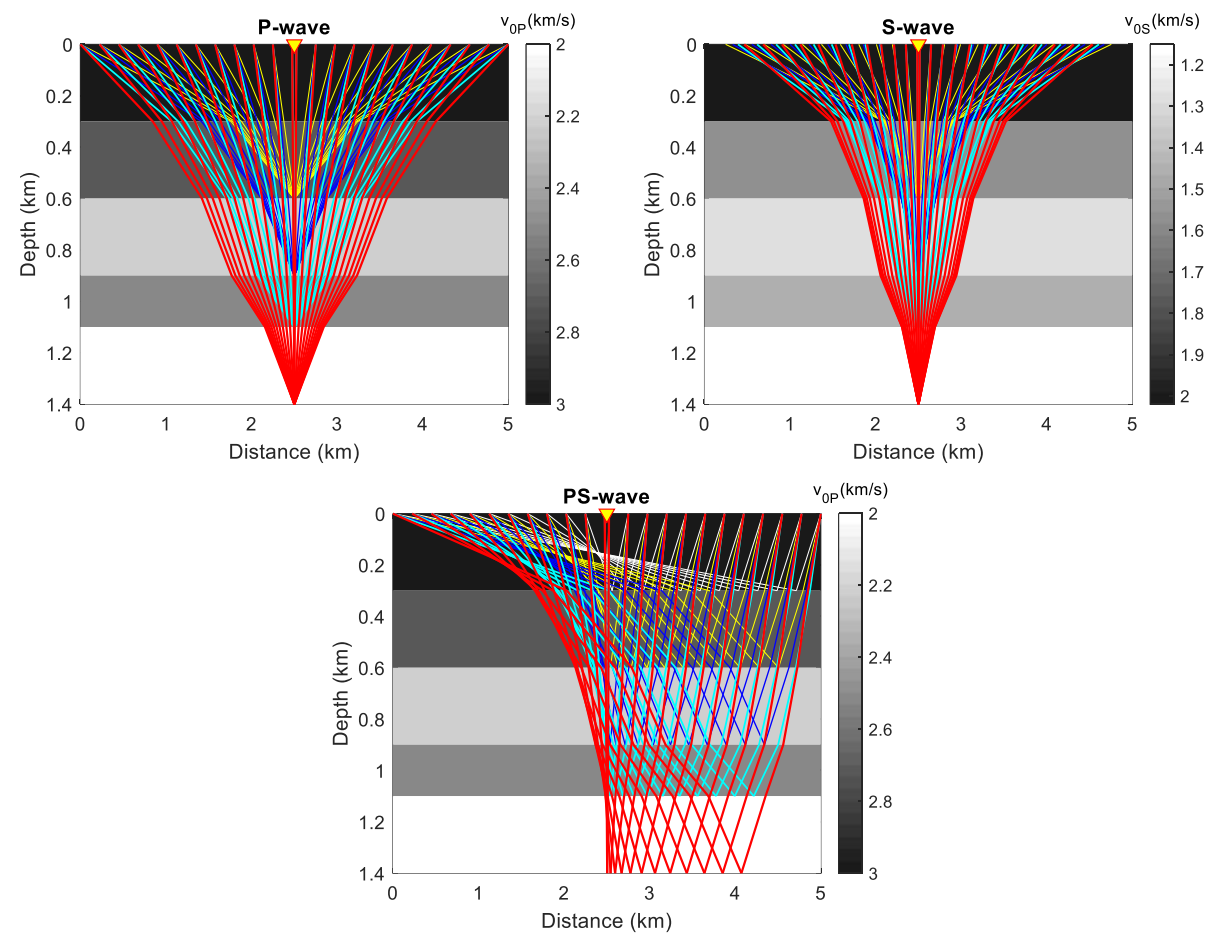

Figure 1. Computed rays for P, S, and converted PS-waves reflections in a CMP gather. A triangle shows the CMP location. The model properties are presented in Table 2.
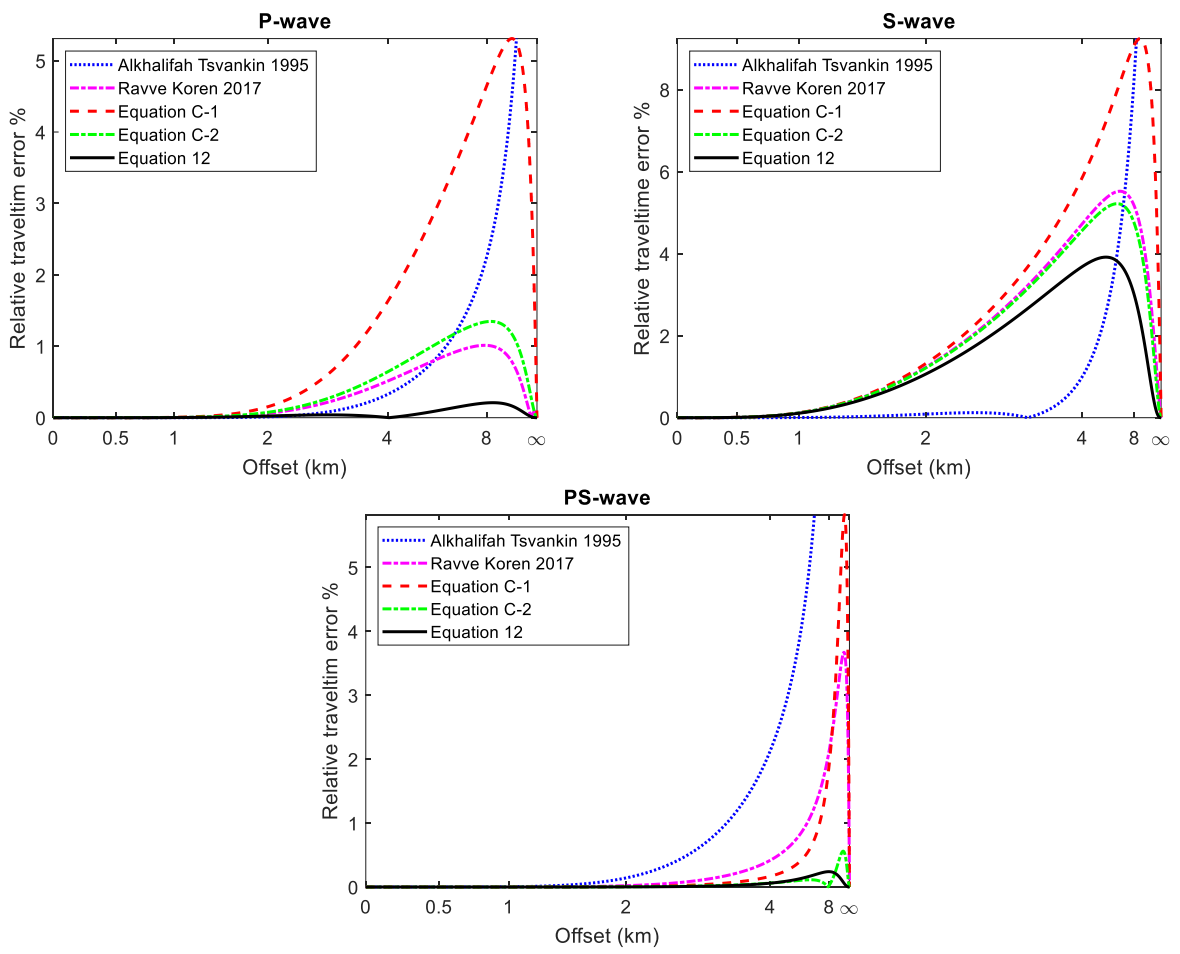

Figure 2. Relative errors of different traveltime approximations for P-, S- and converted PS- waves. We show the results from the classical approximation of Alkhalifah and Tsvankin (1995), Ravve and Koren (2017; equation 57) and the approximations stemming from this work. The horizontal axes use a linear scale in phase angle. 


\section{Elastic approximations for layered media}
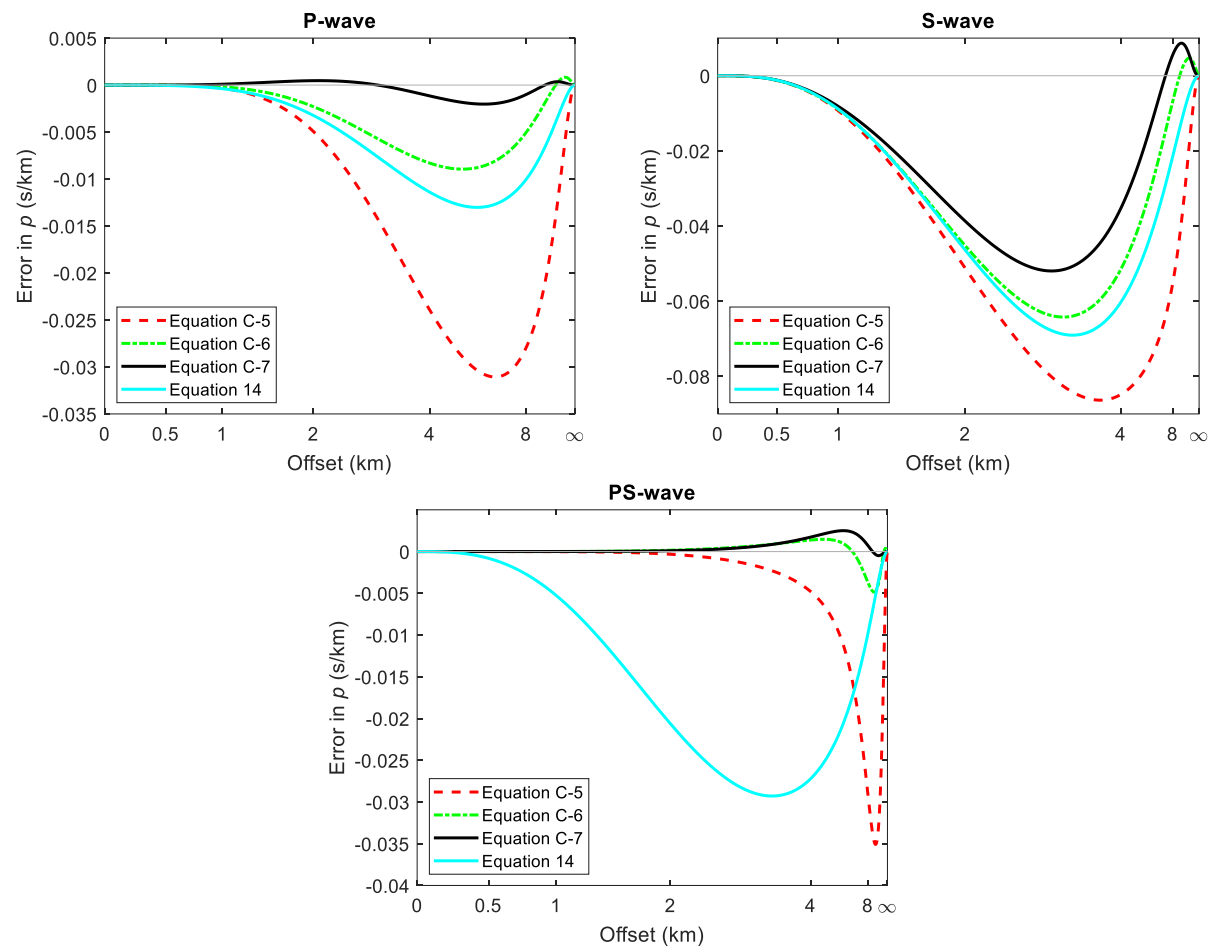

Figure 3. Errors in approximated ray-parameter $(p)$ as a function of offset using the multilayer model in Table 2. The horizontal axes use a linear scale in phase angle.
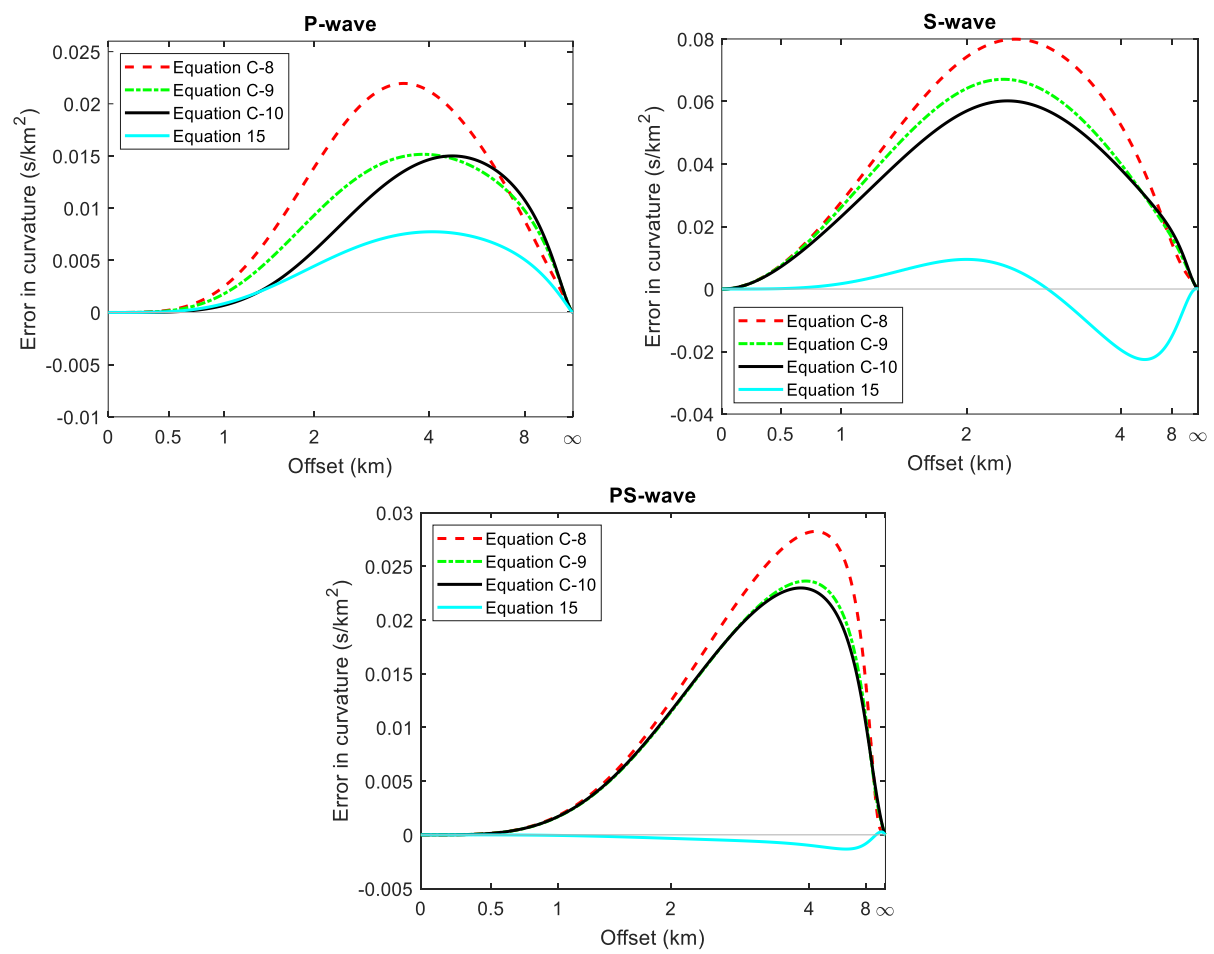

Figure 4. Errors in approximated curvature $(\Psi)$ as a function of offset using the multilayer model in Table 2. The horizontal axes use a linear scale in phase angle. 

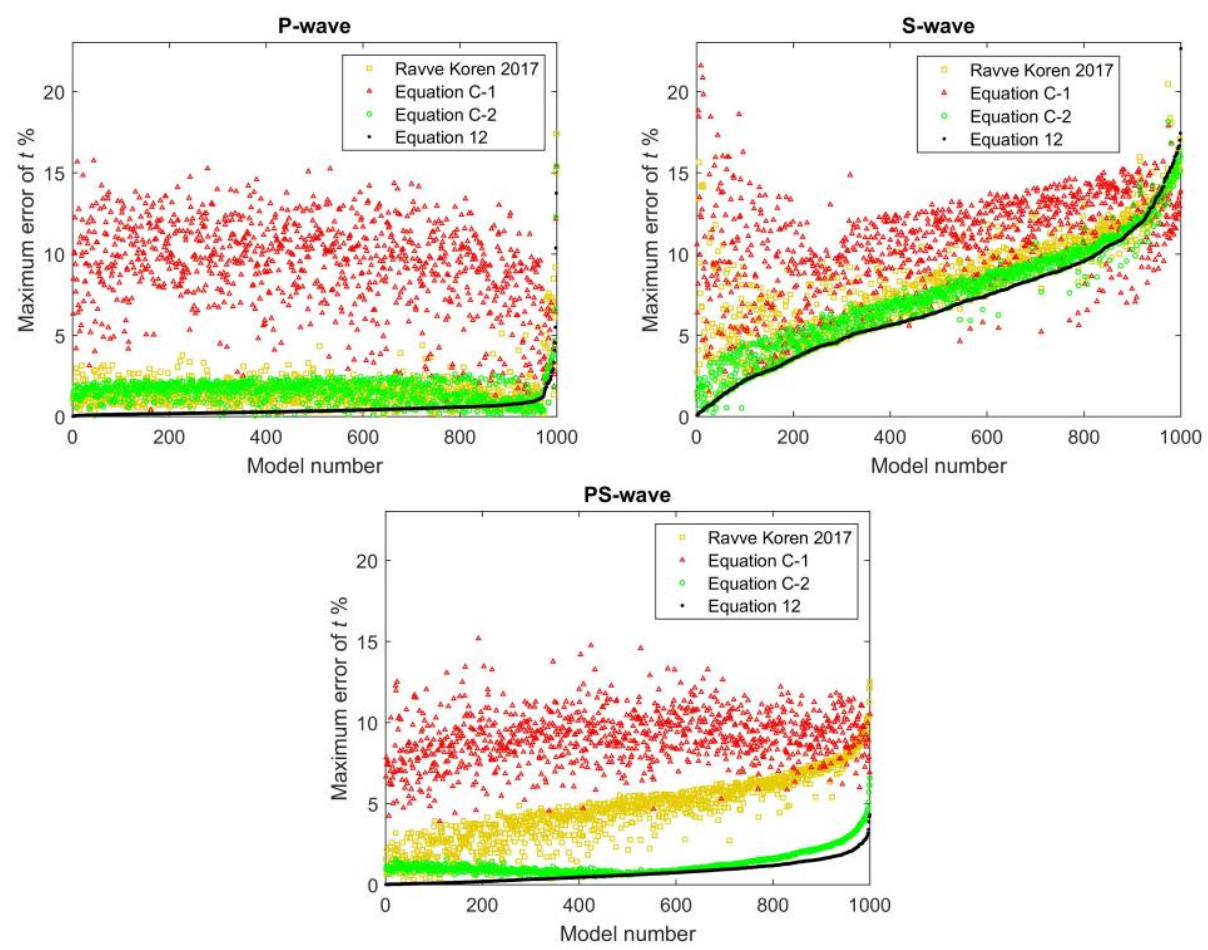

Figure 5. Maximum relative errors of different traveltime approximations for P, S, and PS-waves, corresponding to different randomly generated models. For S-waves, we restrict the models to avoid triplications. In each part, the models are sorted by the errors associated with equation 12 .
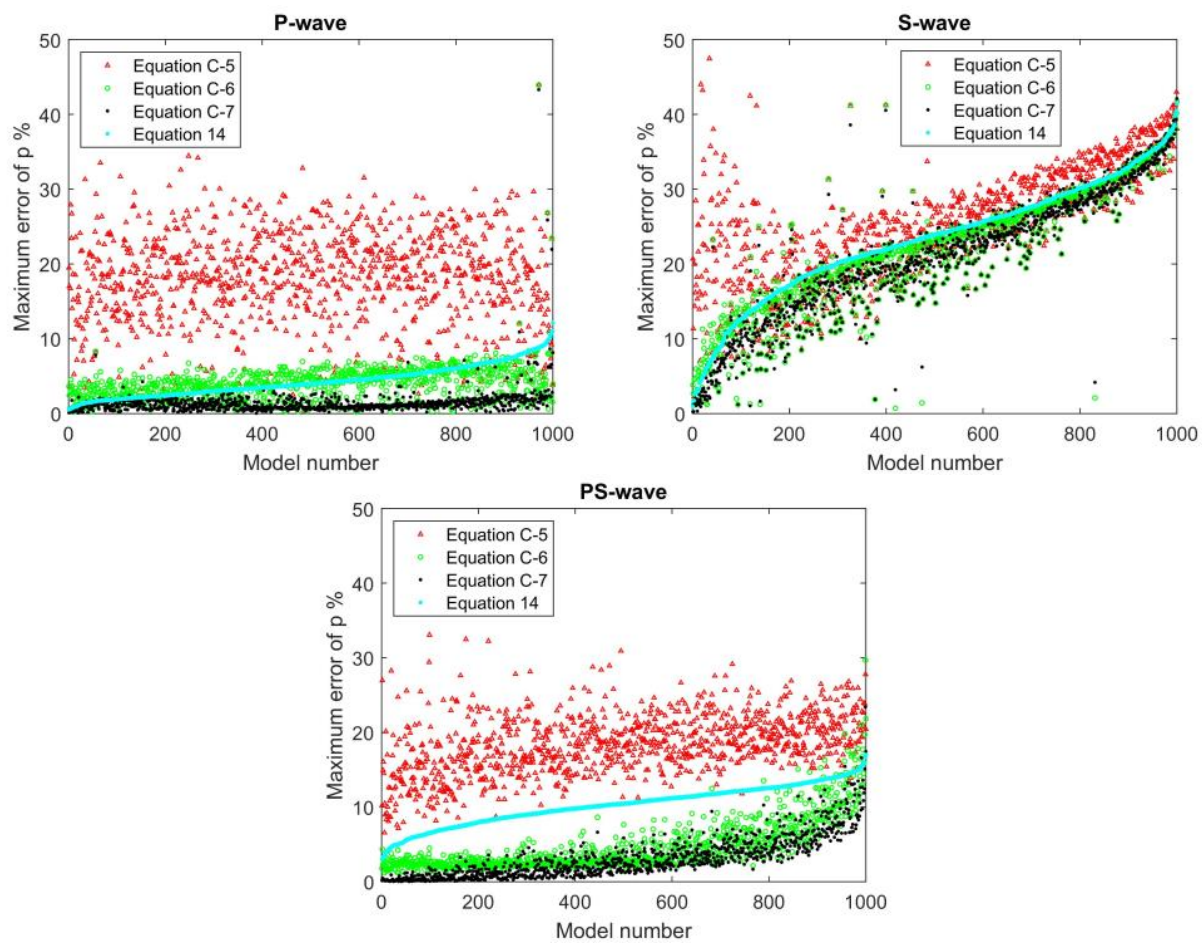

Figure 6. Maximum normalized errors of different ray-parameter approximations for P, S, and PS-waves, corresponding to different randomly generated models. The models are the same as those for Figure 5; however, sorted in each panel by the errors associated with equation 14 . 
Elastic approximations for layered media
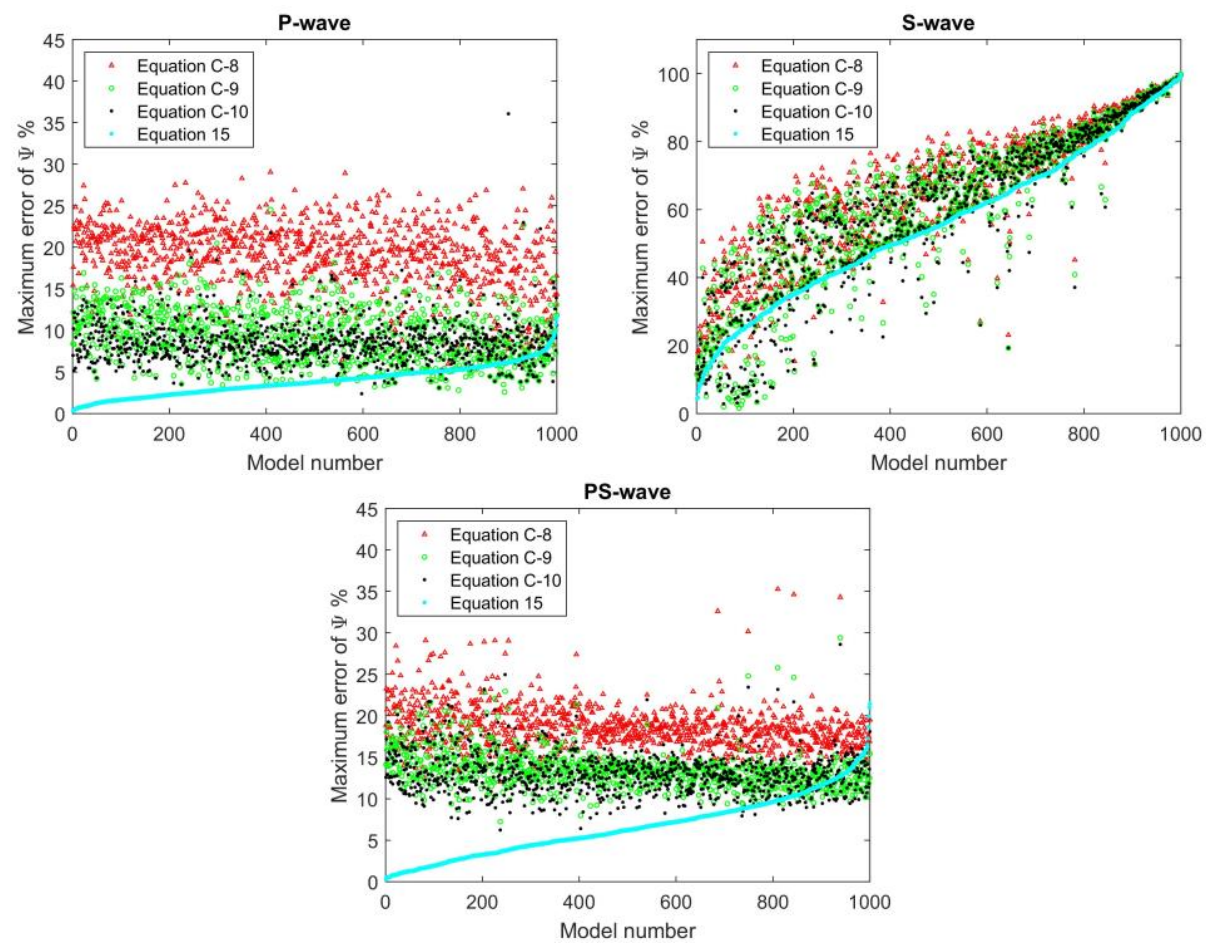

Figure 7. Maximum normalized errors of different curvature approximations for P, S, and PS-waves, corresponding to different randomly generated models. The models are the same as those for Figure 5, but sorted in each panel by the errors associated with equation 15 . 
a)

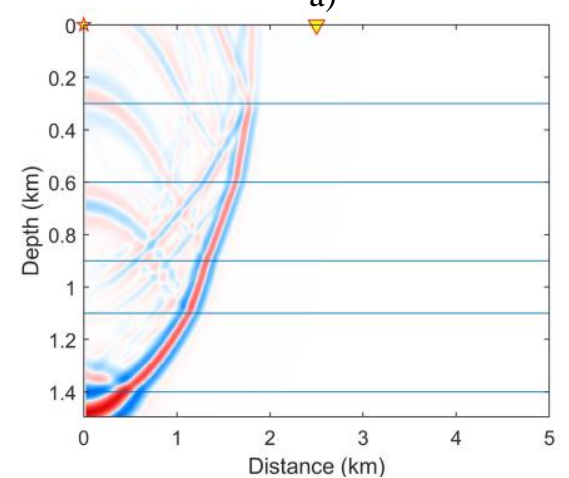

c)

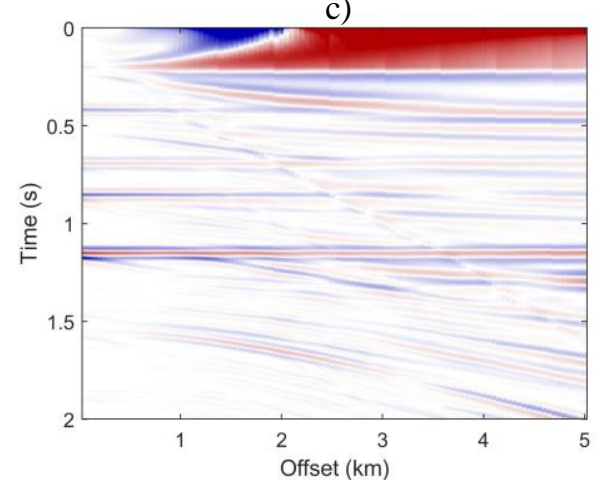

e)

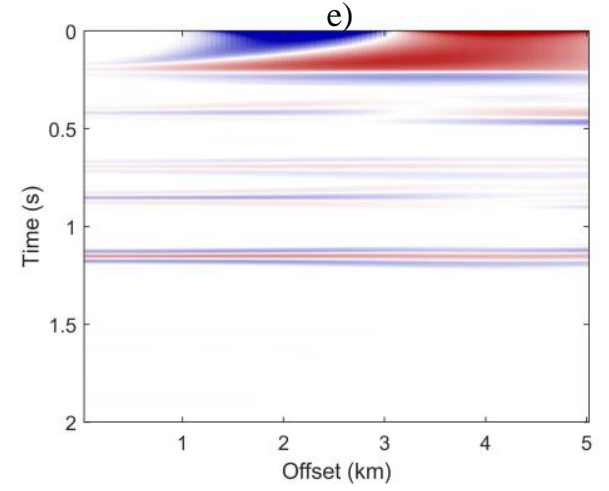

b)

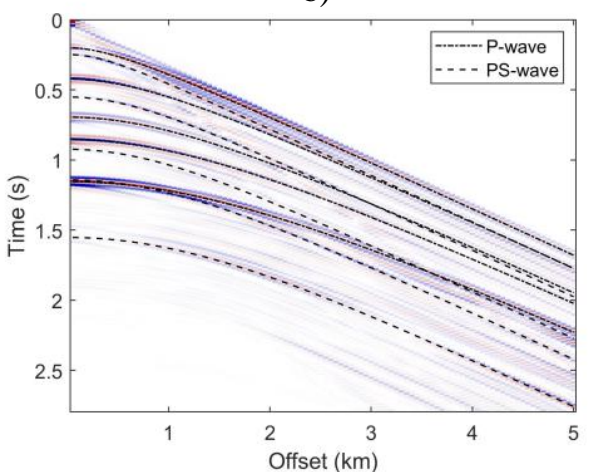

d)

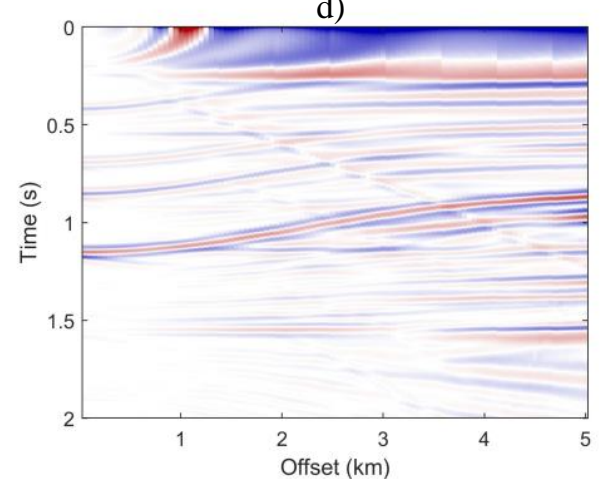

f)

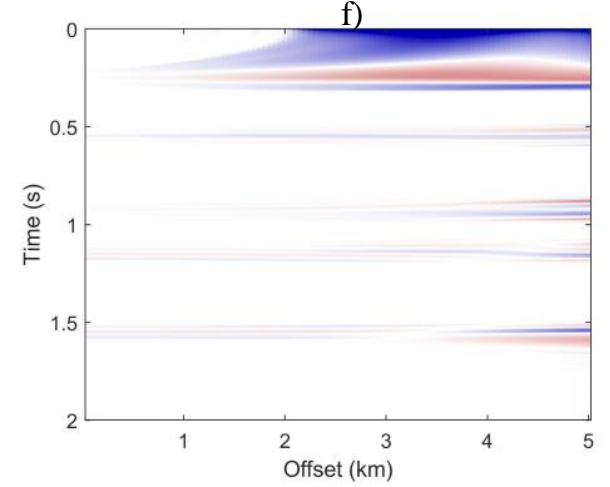

Figure 8. Application of the proposed approximations for $\mathrm{NMO}$ and geometrical spreading correction of $\mathrm{P}$ and PS waves. a) A Snapshot of the finite-difference modeled elastic wavefield corresponding to the multilayer model of Table 2; a star indicates the shot location. b) The modeled CMP gather, overlaid by the traveltimes of the rays shown in Figure 1. c) NMO correction and GS compensation for P-waves data, using equations 12 and 16. d) NMO correction and GS compensation for PS-wave data, using equations 12 and 16. e) Filtering (c) to keep primary P-wave reflections using an anisotropic Radon transform. f) Filtering part (d) to keep primary PSwave reflections using an anisotropic Radon transform. 